Objective To compare mode of delivery in fetuses with known congenital heart disease (CHD) versus the background rate in nonanomalous fetuses.

Methods We examined all cases of prenatally-diagnosed CHD over the 5-year study period, 2007-2011. Data were extracted from computerised patient records. Control data for non-anomalous fetuses were obtained from published hospital records for 2007. Categorical data were analysed using Fisher's exact test (5\% level significant).

Results We identified 242 cases of prenatally-diagnosed CHD over the study period. We excluded 25 lethal karyotypes, 7 miscarriages and 1 termination. Of the remaining 209 cases, complete labour records were available for 158 women. There were 146 live births (92\%) and 12 antepartum stillbirths at $\geq 24$ weeks (8\%). Of the live-born infants with $\mathrm{CHD}$, the perinatal mortality rate was 41 per 1,000. Extra-cardiac defects and non-lethal karyotypic abnormalities were present in $22 \%(n=34)$ and $11 \%(n=18)$ of the cohort respectively. Overall, $23 \%$ (34/146) underwent elective caesarean section (CS). The remaining 112 women had a trial of labour, with a $13 \%(n=15)$ intrapartum CS rate. The rate of intrapartum CS for nulliparous women with known CHD was $18 \%$ (8/45), which was not different to the rate in nulliparous controls in $2007(13 \%$; 432/3324; $p=0.27)$. The equivalent rate in multiparous women was $10 \%(7 / 67)$ in CHD versus $2.4 \%(80 / 3392)$ in controls without previous CS $(\mathrm{p}=0.0013)$.

Conclusions The rate of intrapartum CS in fetuses with known $\mathrm{CHD}$ is not different to the background rate in nulliparous women but is increased in multips.

\section{PL.26 VALIDATION OF A FORMATIVE ASSESSMENT TOOL FOR VACUUM DELIVERY}

doi:10.1136/archdischild-2013-303966.210

'R Bahl, ${ }^{2} \mathrm{DJ}$ Murphy, 'BK Strachan. ' St. Michael's Hsopital, University Hospitals Bristol NHS Trust, Bristol, UK; ${ }^{2}$ Coombe Maternity Hospital, University of Dublin, Dublin, Ireland

Introduction Operative vaginal delivery is one of the first operative procedures a trainee obstetrician performs independently. Competence is developed through work place experience and formative feedback in the form of OSATS. In OSATS each subtask is classified as 'competent' or 'needs help' with a global rating scale to support the assessment. We have developed a formative assessment tool that is likely to aid the feedback by classifying each subtask into five skill levels.

Aim To ascertain the construct validity of a new formative assessment tool.

Method Twenty three videos of vacuum delivery conducted by 10 expert (ST6 and above) and 13 novice (ST1 and ST2) obstetricians were recorded. The video recordings were anonymised and reviewed by a senior obstetrician using the formative assessment tool to score the video recording. There were a total of 12 subtasks, each scored between one and five.

Results The mean score for the experts was 48.4 (80.6\%) compared to the mean score for novices of 34.6 (57.7\%). The students $\mathrm{T}$ test result was significant at 5.01 with $\mathrm{p}$ value of $<0.001$ and $95 \%$ confidence intervals of 8.6 to 19.1 . The average median score for experts was 4.8 and for novices was 3.3.

Discussion The above findings show that the formative assessment tool for vacuum extractor delivery has construct validity and is able to differentiate between an expert and a novice. We believe that the detailed feedback using this assessment tool will facilitate greater understanding of the skills required to develop expertise in vacuum extractor delivery.

\section{PL.27 ASSESSMENT OF THE DELIVERY OF CELL PENETRATING PEPTIDES TO HUMAN UTEROPLACENTAL CELLS}

doi:10.1136/archdischild-2013-303966.211

'LRI Gurney, 'M Sweeney, ${ }^{2} \mathrm{~A}$ Jones, ${ }^{1} \mathrm{~S}$ Robson, 'M Taggart. 'Newcastle University, Newcastle Upon Tyne, UK; ${ }^{2}$ Cardiff University, Cardiff, UK
Introduction The increasing incidence of preterm birth, the severity of its consequences and the inability of current therapies to improve morbidity and mortality in clinical trials creates an urgent need to develop effective new treatments ${ }^{1}$. Cell penetrating peptides (CPP's) are short peptides that facilitate delivery of drug cargo across plasma membranes, showing great promise as intracellular drug delivery vectors in many clinical fields ${ }^{2}$. However, the efficacy of CPP delivery of cargo to human uteroplacental cells remains to be resolved. We aimed to explore the capacity of 3 different CPPs to deliver fluorescent cargo to human myometrial and placental cells in vitro.

Methods Human myometrial and amnion cell cultures were prepared from tissues obtained at elective Caesarean section. Three separate CPPs (TAT peptide, polyarginine, and Penetratin peptide) were conjugated to AlexaFLuor488 dye and compared with noncell-permeable peptide $\left((\mathrm{GC})_{4}\right)$. Cells were incubated at $37^{\circ} \mathrm{C}$ with fluorescently labelled CPP-cargo conjugates and visualised using live cell confocal microscopy.

Results Myometrial or amnion-derived cells consistently expressed fluorescent cargo delivered with each CPP at 1-10 $\mu \mathrm{M}$ after 1-4 hours. Peptide staining often was punctuate throughout the cell cytoplasm appearing perinuclear at the longest timepoints and highest concentrations. At concentrations below $1 \mu \mathrm{M}$ there was little evidence of cargo uptake. No fluorescent cargo was delivered with (GC) ${ }_{4}$.

Conclusion CPP's show promise as cargo delivery vectors in human uteroplacental cells. Their potential use as vectors for bioactive cargo in these cells requires further study.

\section{REFERENCES}

1. Goldenberg et al. Epidemiology and causes of preterm birth. Lancet 2008:372 75-84.

2. Orange \& May. Cell penetrating peptide inhibitors of nuclear factor-kappa B. Mol Life Sci 2008;65:3564-3591.

\section{PL.28 WITHDRAWN BY AUTHOR}

\section{PL.29 MOBILE APPS: THE FUTURE OF PREGNANCY PATIENT INFORMATION}

doi:10.1136/archdischild-2013-303966.212

\section{LC Ashelby, R Bahl. St Michael's Hospital, Bristol, UK}

Introduction Throughout a 'normal' pregnancy women receive up to 45 paper-based patient information leaflets from healthcare professionals about pregnancy, birth and postnatal care. At St Michael's Hospital, Bristol we are developing a smart-phone application (app) to deliver our pregnancy related patient information. We aim to improve patient experience whilst significantly reducing financial and environmental costs. There is a paucity of literature around the use of smart phone apps in healthcare, despite this being a growing area commercially. This project assesses the acceptability of women receiving pregnancy related information electronically.

Method A structured answer survey was carried out on 50 consecutive women attending consultant antenatal clinic. The results were collated and analysed.

Results Fifty women participated in the survey. Thirty six (72\%) women owned and used a smart-phone. Of these 21 (58\%) women reported to have used pregnancy related applications on their phones and $33(86 \%)$ stated that they would consider accessing antenatal information through a phone application. 37 (74\%) of women showed a preference towards using applications ( 24 women) or internet (13 women). 19 (38\%) women showed a preference towards using paper leaflets.

Conclusion Women would like their pregnancy patient information in electronic form, and would prefer to use smart phone applications. We anticipate that by the introduction of a smart phone application and providing information on the internet we will improve patients experience whilst reducing costs. 